\title{
Ways for improving the sensing of nano-bio-sensors
}

\author{
Paolo Di Sia ${ }^{1-4}$ \\ ${ }^{1}$ University of Padova, School of Science, School of Engineering, Dept. of Neurosciences, \\ Padova, Italy \\ ${ }^{2}$ Primordial Dynamic Space Research, Verona, Italy \\ ${ }^{3}$ Correspondence: paolo.disia@gmail.com \\ ${ }^{4}$ Webpage: www.paolodisia.com
}

\begin{abstract}
The nano-bio-sensoristic sector is one of the mainstreams of nanotechnology and requires careful information for constant improvement of the nanodevice performance. The sensing/sensitivity plays a peculiar role, is a determinant characteristics, able to cause a great improvement of the device quality. The paper provides a detailed analysis on the casuistry for increasing the performance of nano-systems through enhanced diffusion, studied with the use of a recent analytical transport model, able to accommodate previously not completely understood behaviours and to predict new interesting features at nanoscale.
\end{abstract}

Keywords: Nano-bio-materials; Nano-bio-sensoristics; Device performance; Diffusion; Sensing; Sensitivity; Sustainability; New functional materials; DS model.

\section{Introductory remarks}

The ability to manipulate the matter, together with advances in synthesis and assembly of structures at the nanoscale, is nowadays carrying to important advances in scientific and technological areas. We can think to the discovery and controlled preparation of carbon nanotubes [1], the ability to put engineered molecules on appropriate electrical contacts for measuring the transport characteristics through molecules [2], the availability of proximal probe techniques for the manipulation of matter [3], the development of chemical synthetic methods for preparing nanocrystals [4], the introduction of biomolecules and supermolecular structures for nanodevices [5], the isolation of biological motors and their incorporation into non-biological environments [6].

omissis

In these years it has been performed a new generalization of the DrudeLorentz model, based on the complete Fourier transform of the frequency-dependent complex conductivity of a system, that relies on analytical expressions of the most important quantities related to transport phenomena, i.e. the velocities correlation function at the temperature $T$, the mean squared deviation of position and the diffusion [11-13]. The model is useful both "a priori", for searching new 
characteristics and peculiarities at nano-level, and "a posteriori", for testing existing experimental data. The comparison with existing used models, as Drude-Lorentz and Smith models, has demonstrated a very good fit and is giving also interesting information about new behaviours at nanoscale $[14,15]$.

omissis

\section{Nanowire-based electronics}

Nanowires-based devices are a powerful class of ultrasensitive devices for utilization at chemical, biological, environmental, medical level, covering areas of life sciences and healthcare $[16,17]$. They represent a detection platform for a broad range of biological and chemical species in solution, with a high number of key features, including direct, label-free, real-time electrical signal transduction, ultrahigh sensitivity, fine selectivity $[18,19]$. These devices are powerful in the detection of proteins, viruses, DNA, and have the potential to notably impact on disease diagnosis, genetic screening, drug discovery.

omissis

\section{Technical details}

In the mainstream of modelling, a generalization of the Drude-Lorentz model, based on the complete Fourier transform of the frequency-dependent complex conductivity $\sigma(\omega)$ of a system, ia based on analytical expressions of the most important quantities related to transport phenomena, i.e. the velocities correlation function $\langle\vec{v}(t) \cdot \vec{v}(0)\rangle_{T}$ at the temperature $T$, the mean squared deviation of position $R^{2}(t)=\left\langle[\vec{R}(t)-\vec{R}(0)]^{2}\right\rangle$ and the diffusion coefficient $D(t)$. It has been performed its classical, quantum and relativistic version [11-13].

The classical version has been deeply tested during years and fits very well with existing experimental data; it gives also explanations of the ultra-short times and of high mobility, with which the charges spread in mesoporous systems, of large interest in photocatalitic and photovoltaic systems [21-24]. It demonstrates high generality and is useful even in the study of ions, like mass transfer, and solutions, so as in nanobiosystems, with significant results with porous and cellular materials, so as for biological, medical and nanopiezotronic devices. 
In detail, we focus on the expressions of the diffusion coefficient, of great importance for its link with the sensitivity of nano-bio-devices.

(I) Classical case

$$
D=2\left(\frac{K_{B} T}{m^{*}}\right)\left[\frac{\tau}{\alpha_{R}} \sin \left(\frac{\alpha_{R}}{2} \frac{t}{\tau}\right) \exp \left(-\frac{t}{2 \tau}\right)\right]
$$

with: $\alpha_{R}=\sqrt{4 \tau^{2} \omega_{0}^{2}-1} \in \mathfrak{R}^{+}$

$$
D(t)=\left(\frac{k_{B} T}{m^{*}}\right)\left(\frac{\tau}{\alpha_{I}}\right)\left[\exp \left(-\frac{\left(1-\alpha_{I}\right)}{2} \frac{t}{\tau}\right)-\exp \left(-\frac{\left(1+\alpha_{I}\right)}{2} \frac{t}{\tau}\right)\right]
$$

with: $\alpha_{I}=\sqrt{1-4 \tau^{2} \omega_{0}^{2}} \in(0,1) \subset \mathfrak{R}$

(II) Quantum case

$$
D=2\left(\frac{K_{B} T}{m^{*}}\right) \sum_{i=0}^{n}\left(\left[\frac{f_{i} \tau_{i}}{\alpha_{i R}} \sin \left(\frac{\alpha_{i R}}{2} \frac{t}{\tau_{i}}\right) \exp \left(-\frac{t}{2 \tau_{i}}\right)\right]\right)
$$

with: $\alpha_{i R}=\sqrt{4 \tau_{i}^{2} \omega_{i}^{2}-1} \in \mathfrak{R}^{+}$

$$
D(t)=\left(\frac{k_{B} T}{m^{*}}\right) \sum_{i=0}^{n}\left(\left(\frac{f_{i} \tau_{i}}{\alpha_{i I}}\right)\left[\exp \left(-\frac{\left(1-\alpha_{i I}\right)}{2} \frac{t}{\tau_{i}}\right)-\exp \left(-\frac{\left(1+\alpha_{i I}\right)}{2} \frac{t}{\tau_{i}}\right)\right]\right)
$$

with: $\alpha_{i I}=\sqrt{1-4 \tau_{i}^{2} \omega_{i}^{2}} \in(0,1) \subset \mathfrak{R}$

(III) Relativistic case

$$
D(t)=2\left(\frac{k_{B} T}{m_{0}}\right)\left(\frac{1}{\gamma}\right)\left(\frac{\tau}{\alpha_{R_{\text {rel }}}}\right)\left[\sin \left(\frac{\alpha_{R_{\text {rel }}}}{2 \rho} \frac{t}{\tau}\right) \exp \left(-\frac{t}{2 \tau \rho}\right)\right]
$$

with $\alpha_{R_{\text {rel }}}=\sqrt{4 \gamma \omega_{0}^{2} \tau^{2}-1} \in \mathfrak{R}^{+}$

$$
D(t)=\left(\frac{k_{B} T}{m_{0}}\right)(\tau)\left(\frac{1}{\gamma}\right)\left(\frac{1}{\alpha_{I_{\text {rel }}}}\right)\left[\exp \left(-\frac{\left(1-\alpha_{I_{r e l}}\right)}{2 \rho} \frac{t}{\tau}\right)-\exp \left(-\frac{\left(1+\alpha_{I_{\text {rel }}}\right)}{2 \rho} \frac{t}{\tau}\right)\right]
$$

with $\alpha_{I_{\text {rel }}}=\sqrt{1-4 \gamma \omega_{0}^{2} \tau^{2}} \in(0,1) \subset \Re$

It also holds:

$$
\alpha_{I_{r e l}}=\sqrt{\Delta_{I_{r e l}}}, \alpha_{R_{r e l}}=\sqrt{\Delta_{R_{r e l}}}, \gamma=1 / \sqrt{1-\beta^{2}}, \beta=v / c, \rho=\gamma^{2} \text {. }
$$

$v$ is the speed of carriers, $c$ the speed of light, $k_{B}$ the Boltzmann's constant, $T$ the temperature of the system, $m_{0}$ and $m^{*}$ rest and effective mass respectively, $\tau_{i}$ and $\omega_{i}$ relaxation time and frequency of the $i$-th state respectively, $\omega_{0}$ center frequency. 
We get the classical expressions of diffusion with $f_{i}=1, \tau_{i}=\tau$ and $\omega_{i}=\omega_{0}=$ center frequency.

Equations governed by the parameter $\alpha_{I}$ are a superposition of exponentials; the behaviour of curves is similar to typical Drude-Lorentz behaviour. Equations governed by the parameter $\alpha_{R}$ are a product of an exponential with a sinusoidal function, with typical damped oscillation in time.

The model, called DS model, includes also a gauge factor, which allows its use from sub-pico-level to macro-level. Interesting applications have been performed also for economics, neuro-science, brain processes, nano-medicine [25,26].

The sensitivity, connected to the increase and rapidity of detection, is one of the most important characteristic of a nano-bio-device, and is linked to values and variation of diffusion.

\section{Discussion and results}

Among the most studied nanomaterials to date we have Silicon (Si), Zinc Oxide ( $\mathrm{ZnO})$, Titanium Dioxide $\left(\mathrm{TiO}_{2}\right)$, Gallium Arsenide (GaAs), Carbon Nanotubes (CNTs), Cadmium Telluride (CdTe), Cadmium Sulfide (CdS), Copper Indium Selenide (CIS), Copper Indium Gallium Selenide (CIGS).

If we consider a nanowire as basis element of a nanobiosystem (device), we can perform an interesting analysis related to the diffusion, obtaining results that allow both to confirm experimental known data and to provide intriguing previsions, useful in the improvement of new high efficiency nanobiosystems, nanobiosensors and nanobiodevices.

About the variables influencing the diffusion and therefore the sensitivity of a nanobiosystem, we have:

a) the temperature $T$ of the system, which is directly proportional to $D$;

b) the parameters $\alpha_{I}, \alpha_{R}$, i.e. the values of $\tau$ and $\omega_{0}$; they appear in the arguments of the exponentials/sin functions in equations, therefore their variation affects also with the shape of the diffusion curves;

c) the variation of the effective mass $m^{*}$, which is connected to the physical and chemical treatment of materials, like doping; electronic, optical, photochemical, photoelectrochemical, photocatalytic and photoexcited properties can be tuned by dopants selection for materials engineering [27,28]; 
d) the variation of the chiral vector inscribed in $(n, m)$ indices, reflecting in a variation of $m^{*}[29]$;

e) at quantum level, the possibility to evaluate the weights of each mode and to vary the carrier density $N$, as it holds $\omega_{p_{i}}{ }^{2}=\frac{4 \pi N e^{2}}{m} f_{i}$, with $\omega_{p_{i}}$ plasma frequencies;

f) at relativistic level, the possibility to act on the initial peak in diffusion through a variation of the carriers velocity.

We consider below some examples of application, considering the classic case for simplicity. The analysis starts considering the behaviour of $D$ for two values of $\tau$ and near to the boundary values of the interval of $\alpha_{I}$. We consider the environmental temperature $T=300 \mathrm{~K}$, but it is also possible to study the behaviour as a function of $T$. Figure 1 represents the behaviour of the diffusion in time for Silicon. The considered values are: $\alpha_{I 1}=0.1, \alpha_{I 2}=0.9, \tau_{1}=10^{-12} s, \tau_{2}=10^{-13} s[30]$.

Figure 1. $D$ vs $t$ for $\operatorname{Si}\left(T=300 K ; m_{S i}=1.08 m_{e}\right)$.

We note how the increase of $\tau$, corresponding to a decrease of $\omega_{0}$ for constant $\alpha_{I}$, helps the increase in diffusion; for $\tau$ constant, the variation of $\alpha_{I}$ brings to a variation in diffusion. The parameter $\alpha_{I}$ changes also the form of curves, reflecting in a variation of the $D$ curve shape.

It follows the same study for $\mathrm{ZnO}$ [31] (Figure 2).

Figure 2. $D$ vs $t$ for $\mathrm{ZnO}\left(T=300 K ; m^{*}{ }_{Z n O}=0.24 m_{e}\right)$.

Figures 3-5 refer the same study for $\mathrm{CdS}$, CdTe and GaAs respectively, considering the same values of $T, \tau$ and $\alpha_{I}$ [32-36].

Figure 3. $D$ vs $t$ for $\operatorname{CdS}\left(T=300 K ; m^{*}{ }_{C d S}=0.22 m_{e}\right)$.

Figure 4. $D$ vs $t$ for $\operatorname{CdTe}\left(T=300 K ; m^{*}{ }_{C d T e}=0.115 m_{e}\right)$.

Figure 5. $D$ vs $t$ for GaAs $\left(T=300 K ; m_{G a A s}^{*}=0.067 m_{e}\right)$.

Figure 6 illustrates the behaviour of diffusion for CNTs with variation of the system temperature; it is $m^{*}=0.5 m_{e}$ [29] ( $m_{e}$ is the electron mass), $\tau=0.17 \cdot 10^{-12} \mathrm{~s}$ $[37,38]$ and $\alpha_{I}=0.5$.

Figure 6. $D$ vs $t$ for CTNs with three different temperatures.

In Figure 7 we compare the value of diffusion of three nanomaterials: CNTs, 
Si and $\mathrm{ZnO}$.

Figure 7. $D$ vs $t$ for three different nanomaterials.

In Figure 8 we focus on the diffusion behaviour of CNTs considering the change of the effective mass as variation of the chiral vector $C_{h}=(n, m)$. We consider CNTs with $(n, m)$ indices equal to $(3,1),(4,2)$ and $(9,2)$. As expected considering the theory, the decrease of the effective mass involves a raise in diffusion.

Figure 8. $D$ vs $t$ for CTNs with three different values of chiral vector $C_{h}=(n, m)$.

We note how the parameter $\alpha$ changes the form of curves, bringing to a shape variation of the diffusion curve [39].

\section{Conclusion}

In this work we have considered a fruitful analysis related to the possibility of varying the carriers diffusion inside a nanostructure. The diffusion is strictly connected to the sensitivity and then to the performance of a nano-bio-system, implying the possibility to get, through a theoretical study, the peculiar characteristics of a nanobiomaterial-based device.

Modelling helps in this direction, giving detailed information about the dynamics of carrier transport from sub-pico-level to macro-level. Acting on chemical, physical, structural and model-intrinsic parameters, i.e the temperature $T$ of the system, the model intrinsic parameters, the values of $\tau_{i}$ and $\omega_{i}$, the variation of the effective mass $m^{*}$, the variation of the chiral vector $C_{h}=(n, m)$, the quantum weights of each mode in the quantum case, the carrier density $N$, the velocity of carriers, it is possible to carry out an accurate fine tuning of the transport dynamics and thus to assess the performance of a nano-bio-device.

Modelling gives new newsworthy innovative informations, precious both for operating devices and in the design phase of planned nano-bio-devices.

The considered nanomaterials can fulfill, through appropriate combinations of the indicated parameters, a high spectrum of practical and technological needs. 


\section{References}

1. Martel, R.; Schmidt, T.; Shea, H.R.; Hertel T.; Avouris, P. Single- and multi-wall carbon nanotube field effect transistors. Appl. Phys. Lett. 1998, 73(17), 2447-2449.

2. Porath, D.; Bezryadin, A.; de Vries, S.; Dekker, C. Direct measurement of electrical transport through DNA molecules. Nature 2000, 403, 635-638.

3. Brukman, M.J.; Bonnell, D.A. Probing Physical Properties at the Nanoscale. Phys. Today 2008, 61, 36-42.

4. Lin, N.; Huang, J.; Chang, P.R.; Anderson, D.P.; Yu, J. Preparation, Modification, and Application of Starch Nanocrystals in Nanomaterials: A Review. J. Nanomater. 2011, 1-13.

5. Hess, H.; Bachand, G.D.; Vogel, V. Powering Nanodevices with Biomolecular Motors. Chem. Eur. J. 2004, 10, 2110-2116.

6. Goel, A.; Vogel, V. Harnessing biological motors to engineer systems for nanoscale transport and assembly. Nat. Nanotechnol. 2008, 3, 465-475.

7. Zhang, X.; Guo, Q.; Cui, D. Recent Advances in Nanotechnology Applied to Biosensors. Sensors 2009, 9, 1033-1053.

8. Di Sia, P. Classical and quantum transport processes in nano-bio-structures: a new theoretical model and applications. PhD Thesis, University of Verona, Italy, 2011.

9. Cohen, A.E.; Moerner, W.E. Method for trapping and manipulating nanoscale objects in solution. Appl. Phys. Lett. 2005, 86(9), 093109-3.

10. Patolsky, F.; Lieber, C.M. Nanowire nanosensors. Mater. Today 2005, 8(4), 20-28.

11. Di Sia, P. An Analytical Transport Model for Nanomaterials. J. Comput. Theor. Nanosci. 2011, 8, 84-89.

12. Di Sia, P. An Analytical Transport Model for Nanomaterials: The Quantum Version. J. Comput. Theor. Nanosci. 2012, 9(1), 31-34.

13. Di Sia, P. Relativistic nano-transport and artificial neural networks: details by a new analytical model. Int. J. Artif. Intell. Mechatron. (IJAIM) 2014, 3(3), 96-100.

14. Di Sia, P. Overview of Drude-Lorentz type models and their applications. Nanosc. Syst.: Math. Mod. Th. Appl. 2014, 3, 1-13.

15. Di Sia, P. Nanotechnology between Classical and Quantum Scale: Applications of a new interesting analytical Model. Adv. Sci. Lett. 2012, 17, 82-86.

16. Di Sia, P. Present and Future of Nanotechnologies: Peculiarities, Phenomenology, Theoretical Modelling, Perspectives. Rev. Theor. Sci. (RITS) 2014, 2, 1-35.

17. Stine, R.; Robinson, J.T.; Sheehan, P.E.; Tamanaha, C.R. Real-Time DNA Detection Using Reduced Graphene Oxide Field Effect Transistors. Adv. Mater. 2010, 22(46), 5297-5300.

18. Gao, A.; Lu, N.; Wang, Y.; Dai, P.; Li, T.; Gao, X.; Wang, Y.; Fan, C. Enhanced Sensing of Nucleic Acids with Silicon Nanowire Field Effect Transistor Biosensors. Nano Letters 2012, 12(10), 5262-5268.

19. Chen, K.-I.; Li, B.-R.; Chen, Y.-T. Silicon nanowire field-effect transistor-based biosensors for biomedical diagnosis and cellular recording investigation. Nanotoday 2011, 6(2), 131-154.

20. Stegh, A.H. Toward personalized cancer nanomedicine - past, present, and future. Integr. Biology 2013, 5, 48-65.

21. Grätzel, M.; Solar energy conversion by dye-sensitized photovoltaic cells. Inorg. Chem. 2005, 44(20), 6841-51.

22. Grätzel, M.; Dye-sensitized solar cells. J. Photoch. Photobio. C: Photochem. Rev. 2003, 4, 145153.

23. Di Sia, P.; Dallacasa, V. Anomalous charge transport: a new "time domain" generalization of the Drude model. Plasmonics 2011, 6(1), 99-104.

24. Smith, N.V. Classical generalization of the Drude formula for the optical conductivity. Phys. Rev. B 2001, 64(15), 155106 (6).

25. Di Sia, P. A New Analytical Model for the Analysis of Economic Processes. Theor. Econ. Lett. 2013, 3(4), 245-250.

26. Di Sia, P. Analytical Nano-Modelling for Neuroscience and Cognitive Science. J. Bioinf. Intell. Control 2014, 3(4), 268-272.

27. Chen, X.; Lou, Y.; Dayal, S.; Qiu, X.; Krolicki, R.; Burda, C.; Zhao, C.; Becker, J. Doped semiconductor nanomaterials. J. Nanosci. Nanotechnol. 2005, 5(9),1408-1420.

28. Doped Nanomaterials and Nanodevices. 3-vol. set, Chen W. ed., University of Texas at Arlington, USA, 2010.

29. Marulanda, J.M.; Srivastava, A. Carrier Density and Effective Mass Calculation for carbon Nanotubes. Phys. status solidi b 2008, 245(11), 2558-2562. 
30. Pirozhenko, I.; Lambrecht, A. Influence of slab thickness on the Casimir force. Phys. Rev. A 2008, 77(1), 013811.

31. Baxter, J.B.; Schmuttenmaer, C.A. Conductivity of ZnO Nanowires, Nanoparticles, and Thin Films Using Time-Resolved Terahertz Spectroscopy. J. Phys. Chem. B 2006, 110(50), 2522925239.

32. Wu, Y.; Wadia, C.; Ma, W.; Sadtler, B.; Alivisatos, A.P. Synthesis and Photovoltaic Application of Copper(I) Sulfide Nanocrystals. Nano Letters 2008, 8(8), 2551-2555.

33. Razykov, T.M.; Contreras-Puente, G.; Chornokur, G.C.; Dybjec, M.; Emirov, Y.; Ergashev, B.; Ferekides, C.S.; Hubbimov, A.; Ikramov, B.; Kouchkarov, K.M.; Mathew, X.; Morel, D.; Ostapenko, S.; Sanchez-Meza, E.; Stefanakos, E.; Upadhyaya, H.M.; Vigil-Galan, O.; Vorobiev, Y.V. Structural, photoluminescent and electrical properties of CdTe films with different compositions fabricated by CMBD. Sol. Energy 2009, 83(1), 90-93.

34. Landolt-Börnstein - Group III Condensed Matter, Vol. 44A, New Data and Updates for I-VII, III-V, III-VI and IV-VI Compounds. Roessler U. ed., 2009.

35. Fernandes da Silva, E.C. GaAs: effective-mass parameters, data extract from Landolt-Börnstein III/44A: Semiconductors - New Data and Updates for I-VII, III-V, III-VI and IV-VI Compounds, Springer-Verlag, Germany, 2009.

36. Parkinson, P.; Lloyd-Hughes, J.; Gao, Q.; Tan, H.H.; Jagadish, C.; Johnston, M.B.; Herz, L.M. Transient Terahertz Conductivity of GaAs Nanowires. Nano Letters 2007, 7(7), 2162-2165.

37. Altan, H.; Huang, F.; Federici, J.F.; Lan, A.; Grebel, H. Optical and electronic characteristics of single walled carbon nanotubes and silicon nanoclusters by tetrahertz spectroscopy. J. Appl. Phys. 2004, 96, 6685-6689.

38. Di Sia, P. About the Influence of Temperature in Single-Walled Carbon Nanotubes: Details from a new Drude-Lorentz-like Model. Appl. Surf. Sci. 2013, 275, 384-388.

39. Di Sia, P. Mathematics and Physics for Nanotechnology - Technical Tools and Modelling. Pan Stanford Publishing Pte. Ltd., Singapore, 2019. 